\title{
Atomistic Simulation of Nanoindentation of Ice $I_{h}$
}

\author{
Pedro Antonio Santos-Flórez, Carlos J. Ruestes, and Maurice de Koning* \\ Cite This: https://dx.doi.org/10.1021/acs.jpcc.0c00255 \\ Read Online
}

ABSTRACT: Using molecular dynamics simulations, we study the nanoindentation response of the ice $\mathrm{I}_{h}$ basal surface using two popular water models, namely, the all-atom TIP4P/Ice potential and the coarse-grained $\mathrm{mW}$ model. In particular, we consider two markedly different temperatures at which a quasi-liquid layer (QLL) is or is not present. We discuss loading curves, hardness estimates, deformation mechanisms, and residual imprints, considering the effect of the QLL, indenter size, and penetration rate. At very low temperatures, in the absence of a QLL, both

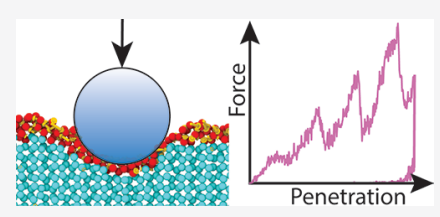
potentials produce similar loading curves and deformation mechanisms. Close to the melting temperature, however, important differences were found, including deviations in the QLL thickness and fraction as well as the presence of a competition between pressure-induced melting and recrystallization events. Nevertheless, both potentials exhibit similar deformation mechanisms and steady-state hardness estimates that are consistent with experimental data. In addition to contributing to the discussion regarding the interpretation of experimental AFM loading curves, the present results provide valuable information concerning the simulation of contact problems involving ice and the behavior of these two popular water models under such circumstances.

\section{INTRODUCTION}

Nanoindentation $^{1,2}$ is an experimental technique that probes mechanical properties of a material by pressing a nanometerscale tip with known mechanical properties into it. With its most common use being elastic modulus and hardness determination in materials, ${ }^{3}$ it has proved to be useful in a wide range of problems in materials science, such as the study of creep, ${ }^{4}$ cracking mechanisms in brittle thin films, ${ }^{5}$ fracture toughness and adhesion assessment on dielectric thin films, ${ }^{6}$ strain hardening and recovery in bulk metallic glasses, ${ }^{7}$ and phase transformations ${ }^{8}$ and even for the study of biological tissues. $^{9}$

Recently, nanoindentation techniques have also been used for "non-technological" materials such as water ice. ${ }^{10,11}$ In particular, atomic force microscopy (AFM) has been employed to probe the ice-vapor surface for the presence of a quasi-liquid layer (QLL), ${ }^{12}$ which is of the utmost importance in several contexts, including glacier flow, environmental processes in clouds, ice adhesion to man-made structures, and even winter sports. These AFM studies ${ }^{13-17}$ focused on various aspects of the QLL; Döppenschmidt and co-workers first performed measurements of its thickness and explored its growth with temperature, $^{13}$ and after which, they assessed the role of impurities, ${ }^{14}$ showing an increase in thickness with the addition of monovalent salt. Butt et al. ${ }^{15}$ analyzed plastic deformation after their AFM curves, estimating the yield strength and investigating the role of the indentation speed and melting rate. They suggested that the liquid-like interfacial layer would be squeezed out by the indenter. Pittenger et al., ${ }^{16}$ in turn, suggest the presence of a QLL at the interface between the tip and ice. They also reported an increase in QLL thickness with increasing temperature. More recently, however, Gelman Constantin et al. ${ }^{17}$ performed AFM studies with different temperature gradients and tip chemistry, reporting QLL thicknesses that are much smaller than those seen in previous AFM studies.

These contradicting findings reveal the difficulty with which the interpretation of such experiments is often met. In this scenario, atomistic simulation techniques can often offer complementary insight, providing a tool for in situ computational "microscopy", 18 capable of providing details of material behavior on atomistic time and length scales. ${ }^{19,20}$ This approach has been particularly useful in the interpretation of nanoindentation experiments for providing atomic-scale details of the processes that occur underneath an indenter tip and are inaccessible experimentally. ${ }^{21-26}$ Nanoindentation simulations for ice, however, have been extremely scarce. To the best of our knowledge, only the study by Gelman Constantin et al. ${ }^{27}$ has reported molecular-level simulations of the indentation process of ice surfaces, investigating the response using the particular TIP5P/Ew model to describe the interactions between water molecules and providing insight into the experimental findings of Pittenger and Butt. Modeling the condensed phases of water has been a notoriously difficult task, however, and oftentimes, the results depend critically on the employed molecular model.

Here, we employ two different water models, namely, the explicit-proton TIP4P/Ice model ${ }^{28}$ and the coarse-grained $\mathrm{mW}$ description $^{29}$ to investigate the response to mechanical

Received: January 10, 2020

Revised: April 1, 2020

Published: April 3, 2020 
indentation by a nanometer-scale tip of the ice $\mathrm{I}_{h}$ basal-plane surface. Furthermore, we employ computational cells that are substantially larger than those considered in ref 27 . Indeed, the cells utilized in the latter are rather thin in the indentation direction, in some cases provoking direct interactions of the indenter tip with both the top and bottom slab surfaces. Not only do our results contribute to the discussion regarding the interpretation of experimental AFM loading curves, ${ }^{11,14-17}$ they provide valuable insight into the behavior of these two popular water models under such circumstances.

\section{COMPUTATIONAL DETAILS}

Water Models and Computational Cells. To describe the interactions between the water molecules, we employ two different approaches, namely, the explicit-molecule TIP4P/Ice model $^{28}$ and the $\mathrm{mW}$ potential ${ }^{29}$ in which the water molecules are treated as "effective atoms" and the protons have been coarse-grained out of the description. A comparison between the results obtained for both force fields provides insight into the role of water's molecular nature and the inherent asymmetry of the hydrogen bonds. . $^{19,30}$

The cells used for the TIP4P/Ice model correspond to defect-free, proton-disordered ice $\mathrm{I}_{h}$ structures with zero total dipole moment. ${ }^{19}$ The cells for the $\mathrm{mW}$ model correspond to the same structures generated for the TIP4P/Ice model but with the protons removed. Most of the simulations presented here were carried out using a small indenter on a cell of $10 \times$ $10 \times 10 \mathrm{~nm}^{3}$ containing 32,032 water molecules with free surfaces on the indentation axis $(z)$ and periodic boundary conditions in the $x$ and $y$ directions. In order to assess the role of the indenter size, a larger cell of $20 \times 20 \times 10 \mathrm{~nm}^{3}$ containing 128,128 molecules was also generated.

Equilibration. The ice cells were first equilibrated at zero pressure and at the desired temperature through an NPT ensemble for which the cell dimensions are allowed to vary independently. In the $x$ and $y$ directions, the pressure is controlled by a barostat, while in the $z$ direction, the pressure is held at zero by introducing a vacuum space in the simulation box above and below the free surfaces. The insertion of empty volume between atom slabs imposes the need to remove dipole interslab interactions so that slab-slab interactions are effectively turned off. ${ }^{31}$ All the simulations have been carried out using the LAMMPS MD package, ${ }^{32}$ a Parrinello-Rahmantype barostat, ${ }^{33-35}$ and a Langevin thermostat. ${ }^{36}$ The corresponding equations of motion are integrated using a time step of $\Delta t=1 \mathrm{fs}$ for the TIP4P/Ice model and $\Delta t=5 \mathrm{fs}$ for the $\mathrm{mW}$ model. For both models, the damping time scales for the thermostat and barostat were chosen as $\tau_{\mathrm{T}}=0.2 \mathrm{ps}$ and $\tau_{\mathrm{p}}=2 \mathrm{ps}$, respectively. For the TIP4P/Ice all-atom model, bond lengths and angles are constrained using the SHAKE algorithm, ${ }^{37}$ and the long-range electrostatics are handled employing the particle-particle-particle-mesh (PPPM) scheme. ${ }^{38}$

Following the initial NPT run, a second equilibration is carried out using boundary conditions that will also be used during the indentation process, as shown in Figure 1. In addition to turning off the barostat, the deepest two bilayers of the cell (see the Supporting Information for definition of the term "bilayer") are now fixed. Furthermore, we also modify the thermostatting scheme, applying a Langevin-thermostat bath only to the two bi-layers above the two fixed layers. In this fashion, the dynamics of the layers that effectively interact with the indenter is governed only by intermolecular forces and the

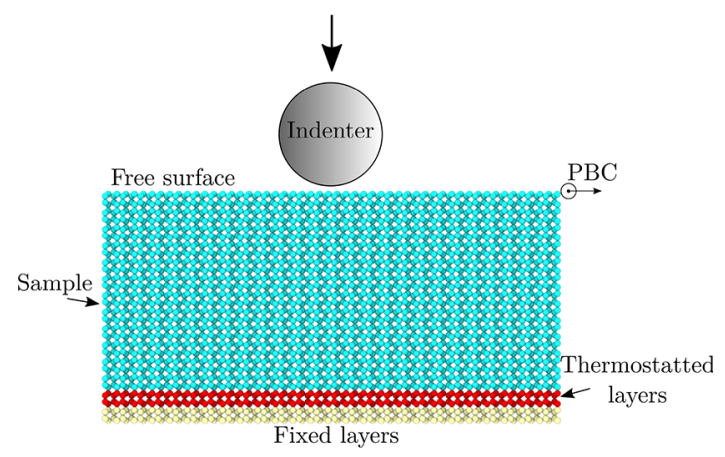

Figure 1. Diagram of the sample geometry and boundary conditions employed for the nanoindentation process using a spherical indenter. Periodic boundary conditions are applied in the two directions perpendicular to the indentation direction. The two molecular layers on the bottom of the cell, shown in yellow, are fixed, whereas thermostatting is applied only to the two subsequent layers, shown in red.

action of the indenter, not being subjected to the artificial dynamical elements introduced by thermostats and barostats. Indeed, this local thermostatting scheme is adequate in that it produces a homogeneous temperature profile for the molecular layers, as illustrated in the Supporting Information.

The simulations have been carried out for two temperatures for both models with the intent of analyzing the influence of a quasi-liquid layer (QLL) at the ice surface. In particular, the indentation simulations have been carried out at a low temperature of $50 \mathrm{~K}$ for which the QLL is absent as well as temperatures of $\sim 5 \mathrm{~K}$ below the melting point when a substantial pre-melt layer is known to cover the ice surface for both models. ${ }^{39-44}$ For a $50 \mathrm{~K}$ temperature, both the first and second equilibration stages involved a time interval of $5 \mathrm{~ns}$. On the other hand, for the temperatures close to the melting point, we increased the length of this interval to $25 \mathrm{~ns}$, given that the equilibration of the QLL is comparatively slow. ${ }^{40}$ This time interval is sufficiently long to produce a converged, steady-state QLL fraction under these conditions, as is further illustrated in the Supporting Information.

Indentation Simulations. Following the second equilibration stage, nanoindentation simulations were performed using the same boundary conditions with a chemically inert indenter characterized by a purely repulsive spherical tip that interacts with the substrate according to the potential-energy expression $^{45}$

$$
V\left(r_{i}\right)= \begin{cases}k\left(R-r_{i}\right)^{3}, & r_{i}<R \\ 0, & r_{i} \geq R\end{cases}
$$

with $R$ as the indenter radius, $r_{i}$ as the distance of molecule $i$ to the center of the indenter, and $k$ as the specified force constant. Given the purely repulsive nature of the indenter-substrate interaction, no capillarity effects are present. The determination of the load-displacement curves is straightforward as the total normal force exerted by the indenter is determined by summing the pairwise $z$-axis contributions of all the molecules interacting with the indenter. All indentation processes were carried out by pressing the indenter into the substrate in a displacement-controlled manner ${ }^{45}$ characterized by a constant indenter velocity using values of 1 and $10 \mathrm{~m} / \mathrm{s}$. To assess possible effects associated with the radius of the indenter we carry out simulations using radii of $R=2 \mathrm{~nm}$ and $R=3 \mathrm{~nm}$. To 
reduce periodic image effects during the indentation, we employ cells with different cross-sectional areas for the two indenter radii using the $10 \times 10 \times 10 \mathrm{~nm}^{3}$ cells for the $2 \mathrm{~nm}$ tip and the $20 \times 20 \times 10 \mathrm{~nm}^{3}$ samples for the $3 \mathrm{~nm}$ radius. Given the prohibitively elevated computational cost associated with the long-range electrostatic interactions of the TIP4P/Ice model, the calculations with the largest tip radius have been done only for the $\mathrm{mW}$ model. To mitigate strain rate effects and study residual imprints, a subset of simulations was performed in three stages. In stage I (penetration stage), the indenter penetrates into the sample to a prescribed depth. Subsequently, in stage II (holding stage), the indenter is kept at constant depth for a given time followed by stage III (removal stage) in which the indenter is removed from the substrate using the same speed used in stage I.

\section{RESULTS AND DISCUSSION}

Sample Equilibration. Before initiating the indentation process, we first assess the equilibration of the computational cells, in particular for temperatures close to the melting point when premelting is known to occur and the formation of a quasi-liquid layer (QLL) on top of an exposed ice surface is established. To analyze the formation of the QLL on the exposed basal plane surface, we monitor the evolution of the number of liquid-like molecules during the equilibration. To this end, we use the Identify-diamond-structure ${ }^{46}$ (IDS) order parameter implemented in the OVITO visualization package to analyze the structure of the oxygen-atom configurations. ${ }^{47}$ For the ice $I_{h}$ structure, it is able to classify particles as of the hexagonal-diamond type as well as its first and second neighbors. We classify as liquid-like those molecules whose oxygen atoms do not belong to any of these structural categories. The advantage of IDS is that, even in situations of symmetry breaking such as at a surface, it classifies all particles as crystalline when no QLL is present at low temperatures. Further details are given in the Supporting Information.

Figure 2 displays snapshots from the 32,032-molecule cells of the molecular structure of the basal plane for the $\mathrm{mW}$

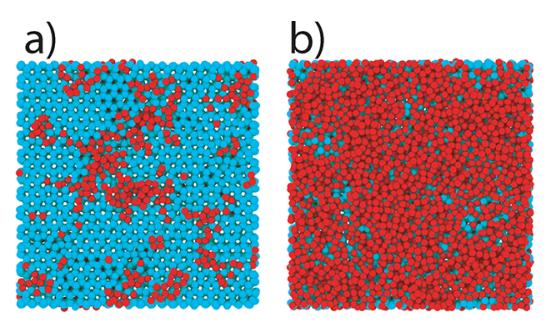

Figure 2. Premelting at the ice-vacuum interface at 269 and $264 \mathrm{~K}$ from simulations using (a) the $\mathrm{mW}$ and (b) TIP4P/Ice water models. Red and cyan spheres depict water molecules in the QLL and hexagonal ice structure, respectively. The fractions of liquid water in the top layer for the $\mathrm{mW}$ and TIP4P/Ice models are $\sim 0.24$ and $\sim 0.83$. respectively.

(Figure 2a) and TIP4P/Ice (Figure 2b) models after equilibration at temperatures of 269 and $264 \mathrm{~K}$, respectively. For the TIP4P/Ice force field, there is a well-formed QLL with a thickness of $\sim 7.5 \AA$, which corresponds to roughly two crystalline layers along the $c$ axis. In addition, the fraction of liquid-like molecules in these layers is approximately 0.83 , which is consistent with previous reports. ${ }^{39-41}$ On the other hand, for the $\mathrm{mW}$ model, the QLL is substantially thinner, showing a layer with a thickness of $\sim 3.7 \AA$, corresponding to a single crystalline bilayer (see the Supporting Information), and a liquid fraction of only $\sim 0.24$. This result deviates from recent studies $^{42-44}$ in which liquid fractions of $\sim 0.65$ were reported. To rule out a possible insufficiency in the equilibration time, we executed a single longer simulation of $100 \mathrm{~ns}$, obtaining the same QLL fraction. A possible reason for this difference is that refs 42-44 all use the CHILL+ order parameter ${ }^{48}$ instead of IDS. As shown in the Supporting Information, in contrast to IDS, the CHILL+ order parameter does not recognize water molecules at a defect-free ice surface to be crystalline even at very low temperatures when no QLL is present.

Loading Curves and Deformation Mechanisms. To choose a value for the indenter force constant $k$, we execute a number of indentation simulations for the 32,032-molecule cell interacting through the $\mathrm{mW}$ model at $T=50 \mathrm{~K}$ using the indenter with $R=2 \mathrm{~nm}$ and a penetration velocity of $1 \mathrm{~m} / \mathrm{s}$. Following previous indentation simulations, we determine the normal force on the indenter as a function of the penetration depth for four different values of $k$ chosen between 1 and 15 $\mathrm{eV} / \AA^{3}{ }^{20,45,49,50}$ As shown in Figure 3, the resulting curves are

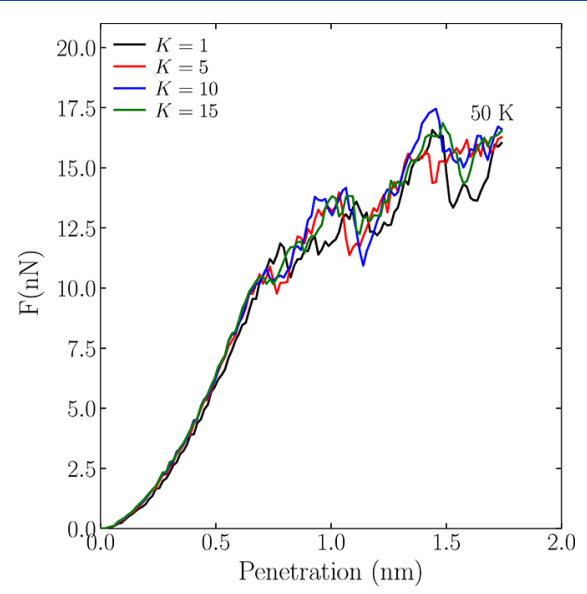

Figure 3. Normal force on the indenter as a function of the indentation depth for the 32,032-molecule cell interacting through the $\mathrm{mW}$ model at $T=50 \mathrm{~K}$ using the indenter with $R=2 \mathrm{~nm}$ and a penetration velocity of $1 \mathrm{~m} / \mathrm{s}$ for four different values of $k$ ranging from 1 to $15 \mathrm{eV} / \AA^{3}$.

essentially insensitive to the particular choice of $k$, indicating that, for these values, the indenter may be considered to be ideally rigid. All remaining indentation simulations have been carried out using $k=10 \mathrm{eV} / \AA^{3}$.

Figure 4 compares typical force-penetration (FP) curves obtained for the TIP4P/Ice and mW models at the low and high temperatures using the $2 \mathrm{~nm}$ indenter tip at a penetration velocity of $1 \mathrm{~m} / \mathrm{s}$. Considering the results obtained for the $\mathrm{mW}$ model, the $50 \mathrm{~K}$ FP curve can be divided into a comparatively smooth elastic regime followed by the occurrence of a succession of stress-relief events during which the normal force on the indenter tip decreases suddenly upon increasing penetration. The first force drop appears at a depth of $\sim 7 \AA$ between the instants marked as A and B in Figure 4. To correlate the non-monotonic features in the FP curve with changes in the substrate microstructure, we analyze atomistic configurations for these two instants and use the hexagonaldiamond structure order parameter ${ }^{46}$ in OVITO to classify the crystalline environments of each water molecule. The results are presented in Figure 5 with the cyan color indicating Wurtzite-type crystallinity while the red color indicates 


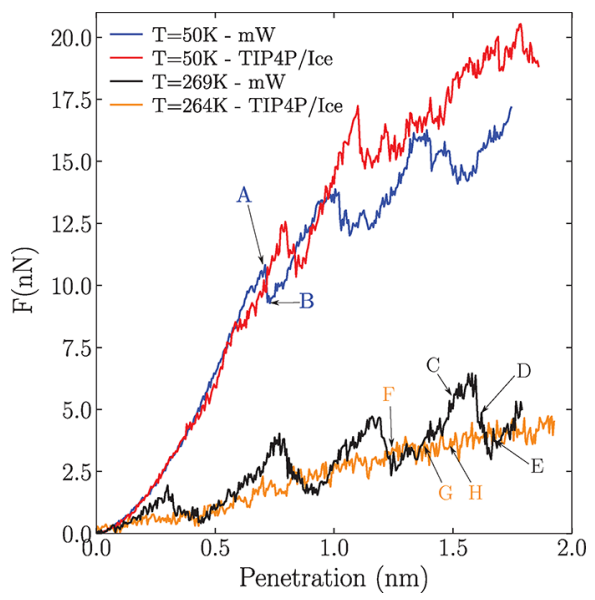

Figure 4. Normal force vs indentation depth during the indentation process, comparing the TIP4P/Ice and $\mathrm{mW}$ models for the two temperatures in study with a tip radius of $2 \mathrm{~nm}$ and a velocity penetration $v=1 \mathrm{~m} / \mathrm{s}$. Letters indicate moments immediately before and after large drops in the FP curves due to localized events, as discussed in the text. Distances between the peaks for the $\mathrm{mW}$ model at $269 \mathrm{~K}$ are close to $3.7 \mathrm{~nm}$, which correspond to the separation between basal-plane bi-layers in the ice $I_{h}$ structure (see the Supporting Information).

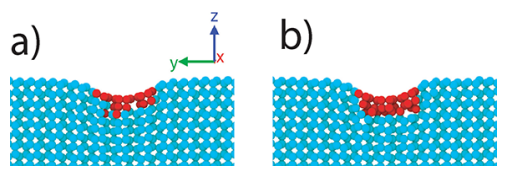

Figure 5. Atomic configurations in (a) and (b) correspond to the instants $A$ and $B$ marked in Figure 4. Particle colors indicate different crystalline environments with cyan indicating particles in a Wurtzitestructure surrounding, including either a Wurtzite first or second neighbor, and red particles belonging to disordered regions.

amorphous structure. As shown in Figure 5a, at $A$, immediately before the first stress-relief event, the top three bilayers are severely strained with some disorder in the two first layers. At configuration $B$, right after the drop as depicted in Figure $5 b$, the extent of the amorphous region below the indenter tip increases to the first three bilayers. This localized amorphization acts as a stress-relief mechanism for the deeper layers. For instance, after the local amorphization of the first three bilayers, the strained part of the fourth bilayer is able to almost entirely restore its unstrained vertical position. The subsequent drops in the FP curve for $\mathrm{mW}-50 \mathrm{~K}$ correspond to a similar amorphization of the fourth and fifth bilayers. Indeed, the penetration-depth distance between the drops is close to the separation between two bilayers in the ice $\mathrm{I}_{h}$ structure.

The FP curve for the TIP4P/Ice model at the same temperature is qualitatively similar to its $\mathrm{mW}$ counterpart with the elastic portions essentially overlapping and a similar pattern of stress-relief events in the plastic regime. Indeed, the basic deformation mechanism associated with the appreciable force drops is the same as that seen for the $\mathrm{mW}$ model, i.e., localized amorphization of the region underneath the indenter tip.

Close to the melting point, however, the behavior is markedly different for both models. While both display similar elastic softening compared to the $50 \mathrm{~K}$ case for small strains, the FP curves display distinct behavior for larger penetration depths. For the mW model, the FP curve features a serrated nature, characterized by a succession of steep rises followed by sudden drops. These sequences are essentially equidistant, separated by a distance of $\sim 3.7 \AA$, which corresponds to the bilayer separation in the ice $\mathrm{I}_{h}$ structure. For TIP4P/Ice, on the other hand, the FP curve increases steadily with the penetration depth without any pronounced features. Inspection of the configurations reveals that the succession of rises and drops corresponds to consecutive bilayer-by-bilayer melting followed by partial recrystallization. This is illustrated in Figure $6 a-c$, which display the oxygen positions at the
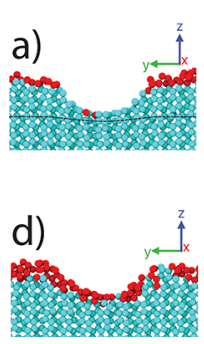
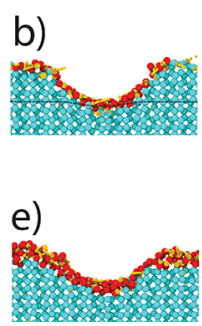

f)

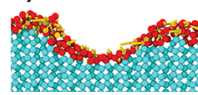

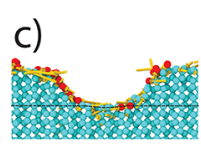

Figure 6. Atomic configurations in $(a-c)$ the top sequence correspond to the instants $\mathrm{C}, \mathrm{D}$, and $\mathrm{E}$ in Figure 4. Atomic configurations in $(d-f)$ the bottom sequence correspond to the instants $\mathrm{F}, \mathrm{G}$, and $\mathrm{H}$ in Figure 4. Particle colors indicate different crystalline environments with cyan indicating particles in a Wurtzitestructure surrounding, including either a Wurtzite first or second neighbor and red particles belonging to disordered regions. Yellow arrows depict the displacements. The dashed black line serves as a guide to the eye to appreciate the relaxation of the tension of the third and fourth bilayers after the melting of the second and third, respectively.

instants marked $\mathrm{C}, \mathrm{D}$, and $\mathrm{E}$ in Figure 4, respectively. At configuration $\mathrm{C}$, close to the end of a rise in the FP curve, the structure underneath the indenter is almost entirely crystalline such that the increase of the FP curve is related to the buildup of elastic strain in the layers below the tip. The subsequent stress drop, illustrated at point $\mathrm{D}$, is achieved by melting of the bilayer immediately below the tip, visibly relieving the strain below. However, after the appearance of the melt layer underneath the indenter, a significant fraction recrystallizes (see Supporting Information), as seen at configuration E, initiating a new buildup of elastic stress. This serrated structure is consistent with the previous findings by Gelman Constantin et al. $^{27}$ using the TIP5P/Ew water model.

The TIP4P/Ice model at $264 \mathrm{~K}$ behaves differently. Although stress relief also occurs by means of bilayer-bybilayer melting, there is no recrystallization, giving an FP curve that is mostly monotonic. As illustrated in Figure 6d-f, which depicts the configurations at the instants marked as F, G, and $\mathrm{H}$ in Figure 4, respectively, the increasing penetration depth gives rise to the same bilayer melting process seen for the $\mathrm{mW}$ model. However, instead of the substantial recrystallization seen for the latter, the indentation pit for TIP4P/Ice remains coated with a liquid-like layer at all times.

Increasing the size of the indenter does not give rise to substantial alterations in the behavior. This is shown in Figure 7, which compares the $2 \mathrm{~nm}$ FP curves to those obtained for an indenter radius of $R=3 \mathrm{~nm}$ for the $\mathrm{mW}$ model. The latter were carried using the large $20 \times 20 \times 10 \mathrm{~nm}^{3}$ cell with double the surface area to reduce image effects due to the larger indenter size. With the exception of the faster rise of the elastic part due to the increased contact area and minor dislocation activity at $50 \mathrm{~K},{ }^{19}$ the general features of the FP curves obtained for both indenter sizes are the same with similar sequences of events 


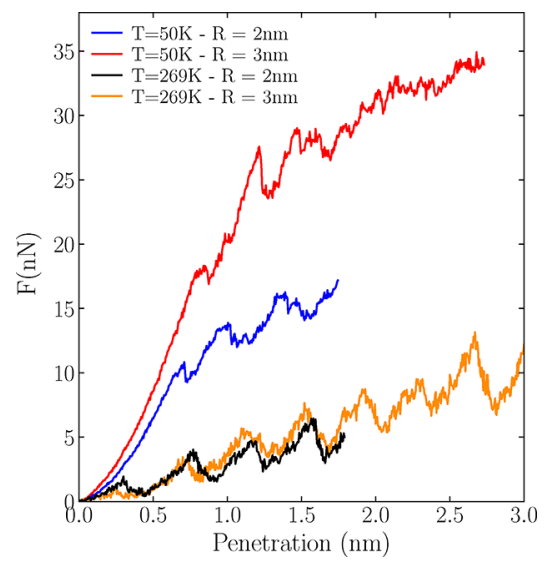

Figure 7. Normal force on the indenter as a function of penetration depth for tip radii of $R=2 \mathrm{~nm}$ and $R=3 \mathrm{~nm}$ for the $\mathrm{mW}$ model at 50 and $269 \mathrm{~K}$ for a penetration velocity of $1 \mathrm{~m} / \mathrm{s}$.

and the activity of the same main relief mechanisms of bilayerby-bilayer amorphization/melting, including its serrated character just below the melting point. The fact that the serrated nature of the FP curve close to the melting point is present for both indenter radii contrasts the findings of Gelman Constantin et al. ${ }^{27}$ in which an indenter radius of 0.55 $\mathrm{nm}$ was found to produce a peak-like structure whereas a value of $1.80 \mathrm{~nm}$ was not. This difference was interpreted as a commensurability issue with the smaller radius being compatible with the bi-layer spacing of $\sim 0.37 \mathrm{~nm}$ whereas the larger tip is not. Our results do not display such compatibility issues, giving serrated FP curves for both the 2 $\mathrm{nm}$ and $3 \mathrm{~nm}$ indenter tips.

Overall, the results above show that, although the main mechanism of stress relief under indentation is the same for both models, namely, bilayer-by-bilayer amorphization/melting, their behavior just below the melting point is very different. While the $\mathrm{mW}$ model displays substantial recrystallization following each melting stress-relief event, leading to a serrated FP curve, the TIP4P/Ice model maintains the presence of a liquid-like layer underneath the indenter at all times. Comparison of these findings to experimental AFM results, ${ }^{13,14,16}$ which indicate the presence of a QLL between the indenter tip and the substrate, ${ }^{13,14,16}$ thus suggests that the explicit-proton TIP4P/Ice model provides a better description of the indentation process compared to the coarse-grained $\mathrm{mW}$ model. This is possibly related to the absence of explicit protons in the $\mathrm{mW}$ model by which the intrinsic asymmetry of the hydrogen bond $(\mathrm{HB})$ with one molecule donating a proton and another accepting it is lost. As such, the formation of effective HBs in this model is not hampered by steric effects, and recrystallization in the presence of a crystalline substrate is facilitated compared to the explicit-proton TIP4P/Ice model. In fact, whether or not HBs are treated explicitly has been shown to fundamentally affect mechanical behavior of ice $\mathrm{I}_{h \cdot} \cdot 19,51$

An indication that this effect is indeed occurring is obtained by reducing the time scale of the indentation process, increasing the tip velocity to $10 \mathrm{~m} / \mathrm{s}$. Figure 8 displays the $\mathrm{mW}$ FP curves obtained with the $3 \mathrm{~nm}$ tip radius at 50 and 269 $\mathrm{K}$ for indenter velocities of 1 and $10 \mathrm{~m} / \mathrm{s}$, respectively. While the behavior is mostly the same for both velocities at $50 \mathrm{~K}$, the increase of the indenter velocity leads to the disappearance of the serrated character of the FP curve at $269 \mathrm{~K}$. This is due to

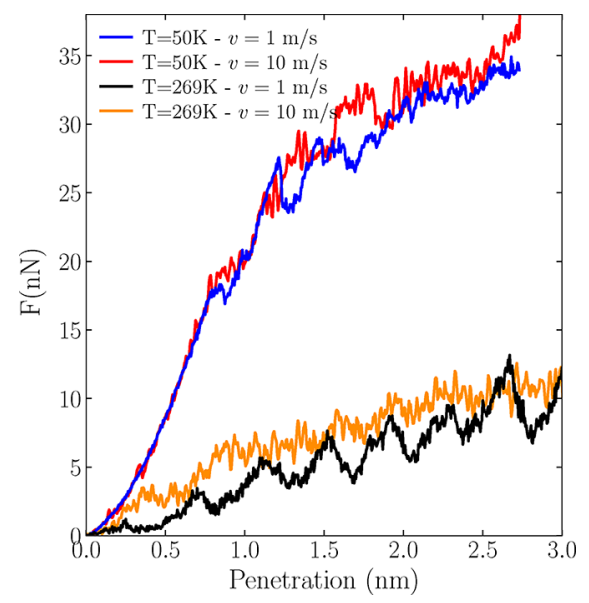

Figure 8. Velocity dependency of the normal force vs indentation depth during the indentation process using the $\mathrm{mW}$ model with a 3 $\mathrm{nm}$ tip radius for the two temperatures in study.

the elevated penetration rate, which prevents liquid atoms below the indenter from recrystallizing, maintaining a QLL between the indenter and bulk ice throughout the entire process.

Hardness. A property that can be estimated quantitatively during the indentation process is the hardness $\mathrm{H}^{2}$ which is related to the contact pressure exerted by the indenter tip. In the Meyer approach, the latter is defined as the ratio between the normal force $F_{\mathrm{N}}$ and the projected contact area $A_{\mathrm{c}}$ of the indenter tip. For a spherical indenter of radius $R$, it is given by the geometric cross-sectional area, $A_{c}=\pi\left(2 R h-h^{2}\right)$, with $h$ as the penetration depth. Figure 9 displays the contact pressure obtained for the TIP4P/Ice and $\mathrm{mW}$ models using the normalforce data from Figure 4.

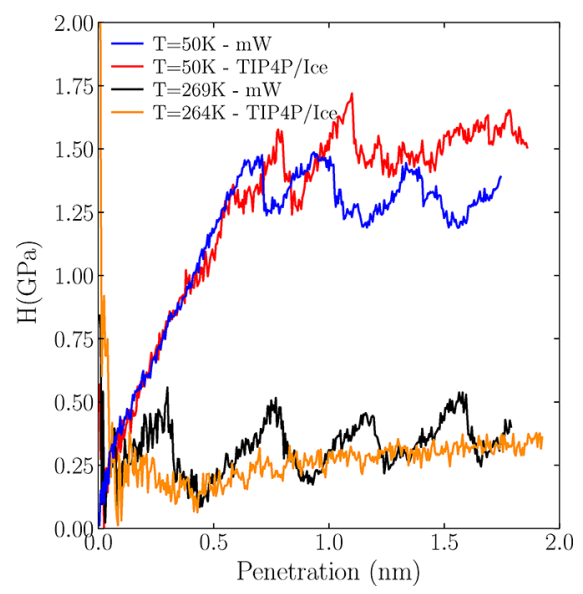

Figure 9. Contact pressure as a function of penetration depth for the $\mathrm{mW}$ and TIP4P/Ice model for the $2 \mathrm{~nm}$ indenter and for the two temperatures in study.

Based on the contact-pressure curve, the hardness can be defined as the average contact pressure after it has reached a steady-state value. For the case shown in Figure 9, this corresponds to the final $1-1.5 \mathrm{~nm}$ of penetration. For the TIP4P/Ice model, the corresponding hardnesses are $H \simeq 1.5$ $\mathrm{GPa}$ and $H \simeq 0.35 \mathrm{GPa}$ for $T=50$ and $264 \mathrm{~K}$, respectively. For the $\mathrm{mW}$ model, the determination of the hardness value is more difficult due to the serrated nature of the contact 
pressure, but taking it to be the average value over the last 1 nm of penetration, we obtain $H \simeq 1.25$ and $H \simeq 0.4 \mathrm{GPa}$ at 50 and $269 \mathrm{~K}$, respectively. In addition to an overall elastic softening of the crystal, the strong decrease of the hardness with temperature is enhanced by the presence of the QLL, in particular for the TIP4P/Ice model.

A direct comparison of these values with experimental estimates is difficult. The main issue is the fact that the values of the hardness measured in this manner depend on the penetration velocities, which, in experiment, are much smaller than those applied in simulations. From a qualitative standpoint, it is known that increasing penetration velocities give rise to higher hardness measures. ${ }^{10,16}$ Our results are consistent with this trend in that the normal-force data for an indenter velocity of $10 \mathrm{~m} / \mathrm{s}$ in Figure 8 give rise to hardness measures that increase by $\sim 10 \%$ for both temperatures. A direct quantitative comparison, however, is inconceivable due to the disparities between the experimental and simulation time scales.

One way to mitigate the effects of the finite penetration rate is to adopt the Meyer estimate of the hardness $H$, which is defined as the average contact pressure with full plasticity developed. ${ }^{2}$ This means that, for a given penetration depth, one measures the contact pressure after the indenter tip has created a stable residual pit with a cross-sectional area close to $A_{\mathrm{c}}$, which persists upon removal of the indenter tip. To this end, we employ a protocol in which, after attaining an indentation depth close to the indenter radius, the position of the tip is fixed, allowing the system to relax during a time interval sufficiently long to attain equilibrium. Subsequently, after reaching a steady value of the contact pressure, the indenter tip is withdrawn with the same velocity used for the indentation stage.

Figure 10 shows the contact pressure as a function of simulation time along the entire loading-unloading cycle for

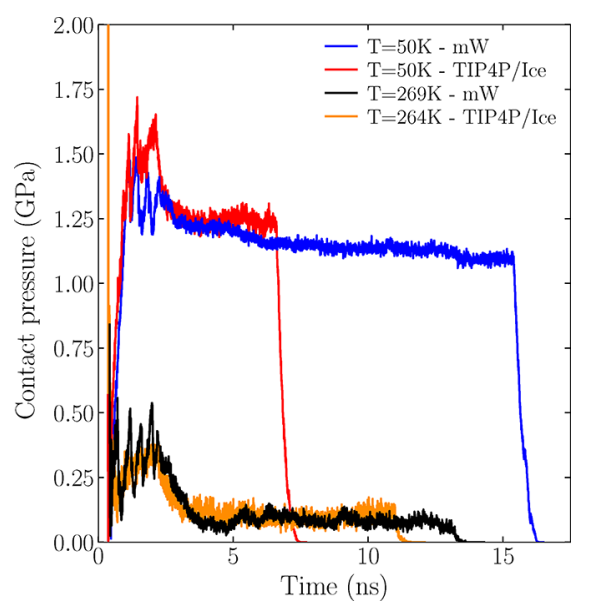

Figure 10. Contact pressure as a function of simulation time during the indentation/hold/withdrawal process for the indenter radius of 2 $\mathrm{nm}$.

the $\mathrm{mW}$ and TIP4P/Ice models for the $2 \mathrm{~nm}$ indenter tip and indentation velocity of $1 \mathrm{~m} / \mathrm{s}$ for both temperatures. The plateau values reached during the hold stage provide the Meyer estimates for $H$. They display very significant reductions compared to their values at the end of the indentation stages, reducing by as much as $\sim 0.30 \mathrm{GPa}$ for the mW model at 269 $\mathrm{K}$. The residual indentation pits after full withdrawal are shown in Figure 11 with well-established amorphous layers for both models at $50 \mathrm{~K}$ and the presence of some QLLs close to the

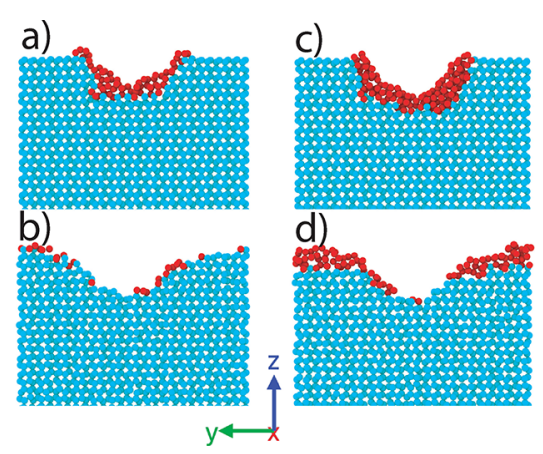

Figure 11. (a, b) Residual structures upon removal of the indenter for the $\mathrm{mW}$ model at 50 and $269 \mathrm{~K}$ are shown, respectively. (c, d) Corresponding images for the TIP4P/Ice model at 50 and $264 \mathrm{~K}$ are displayed, respectively. The color scheme is the same as that in Figure 6a,b.

melting temperature. However, the amount of QLL in the relaxed pit is notably smaller than during the indentation process as seen in Figure 6, in particular for the TIP4P/Ice model. Indeed, when computing the corresponding hardnesses using the relaxed steady-state normal forces, the values for $\mathrm{mW}$ and TIP4P/Ice in the vicinity of their melting temperatures are very close for both models, $\sim 0.08-0.09 \mathrm{GPa}$, of the same order of magnitude as that of the lowest experimental indenter velocity result in $\sim 0.01 \mathrm{GPa}$ as reported by Pittenger et al. ${ }^{16}$ This suggests that, despite the clearly different behavior of the FP curves during the indentation process, in particular, close to their respective melting points, the steady-state hardnesses predicted by both models after reaching stable indentation pits are much less affected by the QLL, which is seen to recede mostly to the surface outside the pits for both models. These findings are consistent with the interpretation of the experiments of Butt et al., ${ }^{15}$ arguing that most of the QLL is squeezed out from the indentation pits and also lends support to the recent experiments of Gelman Constantin et al. ${ }^{17}$ that report substantially reduced QLL thicknesses.

\section{CONCLUSIONS}

In conclusion, we have used molecular dynamics simulations to investigate the response of ice $\mathrm{I}_{h}$ under a nanoindentation process on a [0001]-oriented surface using the all-atom TIP4P/Ice potential and coarse-grained $\mathrm{mW}$ model. Using a purely repulsive, chemically inert spherical tip, we studied the nanoindentation response under controlled displacement conditions for two markedly different temperatures, the first being $50 \mathrm{~K}$ at which the QLL is absent and the second corresponding to a value of $5 \mathrm{~K}$ below the respective melting temperatures for the both models with a substantial QLL presence. Furthermore, we also explore the role of the indenter size and displacement velocity on the loading curves and deformation mechanisms.

We find that the QLL fraction is dramatically different for the two potentials just below the melting temperature; not only does the fraction differ by more than a factor of 3 , there are also substantial differences in the QLL thickness with TIP4P/Ice presenting the largest QLL fraction and thickness.

Overall, the dominating deformation mechanism is the same for both models, namely, bilayer-by-bilayer amorphization/ 
melting induced by pressure. However, while the TIP4P/Ice model maintains the presence of a QLL below the indenter at all times, the $\mathrm{mW}$ model alternates recrystallization and amorphization events, giving rise to serrations in the loading curves for the latter. Such behavior is attributed to the lack of explicit protons in the $\mathrm{mW}$ model, which facilitates recrystallization compared to the TIP4P/Ice model.

Notably, despite differences in the formulation of the two water models used, the obtained results for the steady-state hardness for both models were found to be consistent with experimental AFM reports, giving values that are of the same order of magnitude. Furthermore, in agreement with the experimental literature, hardness increases as the penetration rate grows, while it decreases as temperature increases with an overall softer response attributed to the presence of the QLL.

Residual imprints remaining after removal of the indenter show that the amorphization found for very low temperature is stable and remains in the pits. Just below the melting point, however, the residual imprints have no amorphous layers, and most of the QLL atoms have been displaced to the surface outside the pit, in agreement with the interpretation of Butt et al. in ref 15 and possibly explaining the recent measurements by Gelman Constantin et al. ${ }^{17}$

The results presented here serve a variety of purposes. In addition to contributing to the discussion regarding the interpretation of experimental AFM loading curves, ${ }^{1,14-17}$ they provide valuable information concerning the simulation of contact problems involving ice and the characterization of two popular water models under such circumstances. In particular, future efforts may include a more accurate computational representation of AFM indenter tips in terms of shape/size and their interaction with the substrate ${ }^{52}$ to capture effects such as jump-to-contact phenomena. ${ }^{21}$

\section{ASSOCIATED CONTENT}

\section{(s) Supporting Information}

The Supporting Information is available free of charge at https://pubs.acs.org/doi/10.1021/acs.jpcc.0c00255.

Definition of the term "bi-layer", temperature profiles with the local thermostatting scheme, and details on QLL equilibration, order parameter, and recrystallization (PDF)

\section{AUTHOR INFORMATION}

\section{Corresponding Author}

Maurice de Koning - Instituto de Física Gleb Wataghin and Center for Computing in Engineering \& Sciences, Universidade Estadual de Campinas, UNICAMP, 13083-859 Campinas, São Paulo, Brazil; (1) orcid.org/0000-0002-0035-4170; Phone: +55 (19) 35215478; Email: dekoning@ ifi.unicamp.br

\section{Authors}

Pedro Antonio Santos-Flórez - Instituto de Física Gleb Wataghin, Universidade Estadual de Campinas, UNICAMP, 13083-859 Campinas, São Paulo, Brazil

Carlos J. Ruestes - Instituto Interdisciplinario de Ciencias Básicas, CONICET, Facultad de Ciencias Exactas y Naturales, Universidad Nacional de Cuyo, 5500 Mendoza, Argentina

Complete contact information is available at:

https://pubs.acs.org/10.1021/acs.jpcc.0c00255

\section{Notes}

The authors declare no competing financial interest.

\section{ACKNOWLEDGMENTS}

We acknowledge support from Capes grant no. 88882.329019/ 2019-01 and Fapesp grant nos. 2013/08293-7 and 2016/ 23891-6. We also acknowledge the Unicamp/Santander agreement allocated to the Unicamp's Internationalization Program (res. GR no. 030/2014) for financial support in academic mobility. Part of the simulations were carried out at CCJDR-IFGW-UNICAMP, CENAPAD-SP (Brazil), and TOKO-FCEN-UNCuyo (Argentina).

\section{REFERENCES}

(1) Oliver, W. C.; Pharr, G. M. Measurement of hardness and elastic modulus by instrumented indentation: Advances in understanding and refinements to methodology. J. Mater. Res. 2004, 19, 3-20.

(2) Fischer-Cripps, A. C. Nanoindentation; Mechanical Engineering Series; Springer: New York, 2011.

(3) Gouldstone, A.; Chollacoop, N.; Dao, M.; Li, J.; Minor, A. M.; Shen, Y.-L. Indentation across size scales and disciplines: Recent developments in experimentation and modeling. Acta Mater. 2007, 55, 4015-4039.

(4) Fischer-Cripps, A. C. A simple phenomenological approach to nanoindentation creep. Mater. Sci. Eng., A 2004, 385, 74-82.

(5) Harding, D. S.; Oliver, W. C.; Pharr, G. M. Cracking during nanoindentation and its use in the measurement of fracture toughness. MRS Online Proc. Libr. 1994, 356, 663.

(6) Volinsky, A. A.; Vella, J. B.; Gerberich, W. W. Fracture toughness, adhesion and mechanical properties of low-K dielectric thin films measured by nanoindentation. Thin Solid Films 2003, 429, 201-210.

(7) Yang, B.; Riester, L.; Nieh, T. G. Strain hardening and recovery in a bulk metallic glass under nanoindentation. Scr. Mater. 2006, 54, $1277-1280$.

(8) Domnich, V.; Gogotsi, Y.; Dub, S. Effect of phase transformations on the shape of the unloading curve in the nanoindentation of silicon. Appl. Phys. Lett. 2000, 76, 2214-2216.

(9) Ebenstein, D. M.; Pruitt, L. A. Nanoindentation of biological materials. Nano Today 2006, 1, 26-33.

(10) Li, Y.; Somorjai, G. A. Surface Premelting of Ice. J. Phys. Chem. C 2007, 111, 9631-9637.

(11) Petrenko, V. F. Study of the Surface of Ice, Ice/Solid and Ice/ Liquid Interfaces with Scanning Force Microscopy. J. Phys. Chem. B 1997, 101, 6276-6281.

(12) Slater, B.; Michaelides, A. Surface premelting of water ice. Nat. Rev. Chem. 2019, 3, 172-188.

(13) Döppenschmidt, A.; Kappl, M.; Butt, H.-J. Surface Properties of Ice Studied by Atomic Force Microscopy. J. Phys. Chem. B 1998, 102, 7813-7819.

(14) Döppenschmidt, A.; Butt, H.-J. Measuring the Thickness of the Liquid-like Layer on Ice Surfaces with Atomic Force Microscopy. Langmuir 2000, 16, 6709-6714.

(15) Butt, H.-J.; Döppenschmidt, A.; Hüttl, G.; Müller, E.; Vinogradova, O. I. Analysis of plastic deformation in atomic force microscopy: Application to ice. J. Chem. Phys. 2000, 113, 1194-1203.

(16) Pittenger, B.; Fain, S. C.; Cochran, M. J.; Donev, J. M. K.; Robertson, B. E.; Szuchmacher, A.; Overney, R. M. Premelting at icesolid interfaces studied via velocity-dependent indentation with force microscope tips. Phys. Rev. B 2001, 63, 134102.

(17) Gelman Constantin, J.; Gianetti, M. M.; Longinotti, M. P.; Corti, H. R. The quasi liquid layer of ice revisited: the role of temperature gradients and tip chemistry in AFM studies. Atmos. Chem. Phys. 2018, 18, 14965-14978.

(18) Zepeda-Ruiz, L. A.; Stukowski, A.; Oppelstrup, T.; Bulatov, V. V. Probing the limits of metal plasticity with molecular dynamics simulations. Nature 2017, 550, 492. 
(19) Santos-Flórez, P. A.; Ruestes, C. J.; de Koning, M. Uniaxialdeformation behavior of ice $\mathrm{I}_{h}$ as described by the TIP4P/Ice and $\mathrm{mW}$ water models. J. Chem. Phys. 2018, 149, 164711.

(20) Valencia, F. J.; González, R. I.; Vega, H.; Ruestes, C.; Rogan, J.; Valdivia, J. A.; Bringa, E. M.; Kiwi, M. Mechanical Properties Obtained by Indentation of Hollow Pd Nanoparticles. J. Phys. Chem. C 2018, 122, 25035-25042.

(21) Landman, U.; Luedtke, W. D.; Burnham, N. A.; Colton, R. J. Atomistic mechanisms and dynamics of adhesion, nanoindentation, and fracture. Science 1990, 248, 454-461.

(22) Luan, B.; Robbins, M. O. The breakdown of continuum models for mechanical contacts. Nature 2005, 435, 929.

(23) Szlufarska, I. Atomistic simulations of nanoindentation. Mater. Today 2006, 9, 42-50.

(24) Szlufarska, I.; Chandross, M.; Carpick, R. W. Recent advances in single-asperity nanotribology. J. Phys. D: Appl. Phys. 2008, 41, 123001 .

(25) Ruestes, J. C.; Alhafez, A. I.; Urbassek, M. H. Atomistic Studies of Nanoindentation-A Review of Recent Advances. Crystals 2017, 7, 293.

(26) Chavoshi, S. Z.; Xu, S. Nanoindentation/scratching at finite temperatures: Insights from atomistic-based modeling. Prog. Mater. Sci. 2019, 100, 1-20.

(27) Gelman Constantin, J.; Carignano, M. A.; Corti, H. R.; Szleifer, I. Molecular Dynamics Simulation of Ice Indentation by Model Atomic Force Microscopy Tips. J. Phys. Chem. C 2015, 119, 2711827124.

(28) Abascal, J. L. F.; Sanz, E.; García Fernández, R.; Vega, C. A potential model for the study of ices and amorphous water: TIP4P/ Ice. J. Chem. Phys. 2005, 122, 234511.

(29) Molinero, V.; Moore, E. B. Water Modeled As an Intermediate Element between Carbon and Silicon. J. Phys. Chem. B 2009, 113, $4008-4016$.

(30) Franco Pinheiro Moreira, P. A.; Gomes de Aguiar Veiga, R.; de Koning, M. Elastic constants of ice $\mathrm{I}_{h}$ as described by semi-empirical water models. J. Chem. Phys. 2019, 150, No. 044503.

(31) Yeh, I.-C.; Berkowitz, M. L. Ewald summation for systems with slab geometry. J. Chem. Phys. 1999, 111, 3155-3162.

(32) Plimpton, S. Fast Parallel Algorithms for Short-Range Molecular Dynamics. J. Comput. Phys. 1995, 117, 1-19.

(33) Parrinello, M.; Rahman, A. Polymorphic transitions in single crystals: A new molecular dynamics method. J. Appl. Phys. 1981, 52, $7182-7190$.

(34) Martyna, G. J.; Tobias, D. J.; Klein, M. L. Constant pressure molecular dynamics algorithms. J. Chem. Phys. 1994, 101, 4177-4189.

(35) Shinoda, W.; Shiga, M.; Mikami, M. Rapid estimation of elastic constants by molecular dynamics simulation under constant stress. Phys. Rev. B 2004, 69, 134103.

(36) Schneider, T.; Stoll, E. Molecular-dynamics study of a threedimensional one-component model for distortive phase transitions. Phys. Rev. B 1978, 17, 1302-1322.

(37) Ryckaert, J.-P.; Ciccotti, G.; Berendsen, H. J. C. Numerical integration of the cartesian equations of motion of a system with constraints: molecular dynamics of n-alkanes. J. Comput. Phys. 1977, $23,327-341$.

(38) Hockney, R. W. Computer Simulation Using Particles; Taylor \& Francis Group: New York, 2017.

(39) Conde, M. M.; Vega, C.; Patrykiejew, A. The thickness of a liquid layer on the free surface of ice as obtained from computer simulation. J. Chem. Phys. 2008, 129, No. 014702.

(40) Limmer, D. T.; Chandler, D. Premelting, fluctuations, and coarse-graining of waterice interfaces. J. Chem. Phys. 2014, 141, $18 \mathrm{C} 505$.

(41) Louden, P. B.; Gezelter, J. D. Why is Ice Slippery? Simulations of Shear Viscosity of the Quasi-Liquid Layer on Ice. J. Phys. Chem. Lett. 2018, 9, 3686-3691.

(42) Hudait, A.; Allen, M. T.; Molinero, V. Sink or Swim: Ions and Organics at the Ice-Air Interface. J. Am. Chem. Soc. 2017, 139, 1009510103.
(43) Qiu, Y.; Molinero, V. Why Is It So Difficult to Identify the Onset of Ice Premelting? J. Phys. Chem. Lett. 2018, 9, 5179-5182.

(44) Pickering, I.; Paleico, M.; Sirkin, Y. A. P.; Scherlis, D. A.; Factorovich, M. H. Grand Canonical Investigation of the Quasi Liquid Layer of Ice: Is It Liquid? J. Phys. Chem. B 2018, 122, 48804890.

(45) Kelchner, C. L.; Plimpton, S. J.; Hamilton, J. C. Dislocation nucleation and defect structure during surface indentation. Phys. Rev. B 1998, 58, 11085-11088.

(46) Maras, E.; Trushin, O.; Stukowski, A.; Ala-Nissila, T.; Jónsson, $\mathrm{H}$. Global transition path search for dislocation formation in Ge on Si(001). Comput. Phys. Commun. 2016, 205, 13-21.

(47) Stukowski, A. Visualization and analysis of atomistic simulation data with OVITO-the Open Visualization Tool. Model. Simul. Mater. Sci. Eng. 2010, 18, No. 015012.

(48) Nguyen, A. H.; Molinero, V. Identification of Clathrate Hydrates, Hexagonal Ice, Cubic Ice, and Liquid Water in Simulations: the CHILL+ Algorithm. J. Phys. Chem. B 2015, 119, 9369-9376.

(49) Ruestes, C. J.; Schwen, D.; Millán, E. N.; Aparicio, E.; Bringa, E. M. Mechanical properties of Au foams under nanoindentation. Comp. Mater. Sci. 2018, 147, 154-167.

(50) Ruestes, C. J.; Anders, C.; Bringa, E. M.; Urbassek, H. M. Nanoindentation tests of heavy-ion-irradiated $\mathrm{Au}$ foams-molecular dynamics simulation. J. Appl. Phys. 2018, 123, 225903.

(51) Guo, Q.; Ghaani, M. R.; Nandi, P. K.; English, N. J. PressureInduced Densification of Ice $I_{h}$ under Triaxial Mechanical Compression: Dissociation versus Retention of Crystallinity for Intermediate States in Atomistic and Coarse-Grained Water Models. J. Phys. Chem. Lett. 2018, 9, 5267-5274.

(52) Vishnubhotla, S. B.; Chen, R.; Khanal, S. R.; Hu, X.; Martini, A.; Jacobs, T. D. B. Matching Atomistic Simulations and In Situ Experiments to Investigate the Mechanics of Nanoscale Contact. Tribol. Lett. 2019, 67, 97. 